\title{
Error Function Attack of chaos synchronization based encryption schemes
}

\author{
Xingang Wang, Meng Zhan, C.-H. Lai* \\ Temasek Laboratories, National University of Singapore, \\ 10 Kent Ridge Crescent, Singapore 119260 \\ *Department of Physics, National University of Singapore, Singapore 117542 \\ Hu Gang \\ Department of Physics, Beijing Normal University, Beijing 100875, China
}

\begin{abstract}
Different chaos synchronization based encryption schemes are reviewed and compared from the practical point of view. As an efficient cryptanalysis tool for chaos encryption, a proposal based on the Error Function Attack is presented systematically and used to evaluate system security. We define a quantitative measure (Quality Factor) of the effective applicability of a chaos encryption scheme, which takes into account the security, the encryption speed, and the robustness against channel noise. A comparison is made of several encryption schemes and it is found that a scheme based on one-way coupled chaotic map lattices performs outstandingly well, as judged from Quality Factor.
\end{abstract}

PACS numbers: 05.45.Vx, 05.45.Ra, 43.72.+q 


\section{INTRODUCTION}

Chaotic systems and conventional cryptosystems share many common properties, such as the sensitivity to initial conditions and parameters, high complexity and unpredictability, stochastic and random-like behaviors, and so on [1]. Shanon, in his seminal paper of cryptography, wrote [2]: "In a good mixing transformation ... functions are complicated, involving all variables in a sensitive way. A small variation of any one (variable) changes (the outputs) considerably." These requirements, which are also known as the diffusion and confusion properties in conventional cryptography theory, are just some of the fundamental characteristics of chaotic dynamical systems widely studied in the last several decades. It is indeed a celebrated property of chaotic systems that they can produce highly complex signals while having relatively simple system structures. Whereas the conventional cryptography is mainly based on some particular algebraic or number-theoretic operations, chaos-based cryptography depends entirely on some physical laws, this makes chaos cryptography not only easy to formulate and analyze in theory, but also simply to design and operate in applications. Because of these advantages, chaos encryption has been an extremely popular topic of investigation, pursued in recent years by researchers from different fields.

The conventional cryptography works on discrete-value systems while chaotic cryptography works on continuous-value systems. Because of this, the early attempts in this field mainly focused on using chaotic systems as pseudo-random number $(\mathrm{PN})$ generators in discrete-value implementations 3]. Later, different encryption algorithms were developed based on the different properties of chaos: algorithms that build upon discretization and mapping, ergodicity, perturbation and control, targeting, alphabet units portion etc., have been proposed for different purposes 4]. Each algorithm has its special characteristics and can be well utilized under certain restrictions. In 1990, Pecora and Carroll published the first paper on chaos synchronization and assumed its application in secure communication 5]. From then on, chaos synchronization based encryption (CSE) has been a hot topic both in theory and in applications [6, 7, 8]. Comparing with other algorithms, the CSE algorithm is advanced in many aspects. Firstly, the well developed theory of chaos synchronization provides a solid basis for its feasibility and performance analysis. Secondly, experiments show that chaos synchronization can be robust and easily realizable in applications. Finally, it is easy to synchronize high-dimensional chaotic systems constructed by coupling or cas- 
cading low-dimensional systems, which has been used as a standard method for generating highly complex signals in practical operations. As a result, different encryption schemes based on the idea of CSE, such as chaos masking, chaos key shifting and chaotic modulation etc., have been proposed in recent years.

Initially, chaos encryption was implemented on low-dimensional chaotic systems both theoretically and experimentally. These encryption methods are reasonably fast, simple to implement, and have been assumed secure. However, various security flaws have been found, flaws that allow the private message to be extracted without a knowledge of the secret keys, or of the underlying dynamics $[9,10,11]$. In order to overcome these drawbacks, recent developments have focused on hyperchaos systems which possess many positive Lyapunov exponents (LEs) and are more complex in dynamics. In particular, spatiotemporal chaos has been investigated widely for its excellent performance in correlations and spread spectrum multiple access communication [12]. However, while the early works on chaos encryption were discussed in the community of applied cryptography, recent works are almost exclusively considered inside the nonlinear systems community. Even a casual evaluation of these algorithms shows a lot of potential pitfalls and inherent drawbacks [1, 13]. For a good practical encryption algorithm, security is not the only requirement; other properties such as encryption speed and error rate should be considered as well. Unfortunately, so far in the field of chaos encryption, most efforts are still directed at improving system security, with the other properties receiving little attention. In general, when the dimension of a chaotic system is increased, its encryption speed will suffer, and for a CSE system, the synchronization time will increase too. Together these will effect the system cost and the global performance in different manners. Therefore, it is necessary to analyze different chaos encryption schemes by taking all these crucial aspects into account.

In this paper, we will analyze different chaos synchronization based encryption schemes from the application point of view. For each scheme, three properties: security, encryption speed, and error propagation length (EPL), will be investigated and compared. We propose an efficient cryptanalysis method, the Error Function Attack (EFA) method, for the evaluation of chaos encryption systems. Moreover, we define a quantity, which we call the Quality Factor (QF), that allows us to compare the practical applicabilities of different cryptosystems. Based on the simulation results, we give a rough ranking of various chaos encryption schemes according to their feasibilities for application. 
This paper will be arranged as follows. In Sec. II, we propose and analyze the EFA method in detail. We then evaluate the security of different chaos encryption schemes using the EFA method in Sec. III, and define the Quality Factor. Comparison of the different encryption schemes using the QF is presented in Sec. IV. A brief conclusion is then given in Sec. V.

\section{ERROR FUNCTION ATTACK}

A cryptosystem is usually designed in such a way that its security relies on as few as possible secrets expressed as keys, and it is also believed that a public-structure encryption system is more reliable than a secret one [14]. A fundamental assumption in cryptanalysis enunciated by Kerckhoffs in the nineteenth century is that the security of an algorithm must reside entirely on the key (the Kerckhoffs principle). According to this assumption, for a good encryption algorithm, if Eve (the eavesdropper) wants to extract the message transmitted between Alice (the transmitter) and Bob (the receiver), the only thing she needs to do is try to find out the secret key used; she will not need to spend time on extracting the system's structure. In contrast, most chaos encryption schemes do not observe this principle rigidly and the implementations try to hide everything from the public. This approach has many negative impacts both on the system safety and its commercial applications.

Based on the requirement of public-structure encryption, there are four general types of cryptanalytic attacks, among them the known-plaintext attack is the most common one. In the real world, it is easy for an cryptanalyst to get some past plaintext messages that have been encrypted. Hence, when we evaluate the securities of chaos encryption schemes, vulnerability to the known-plaintext attack should be considered first [14]. In this paper, we will propose a known-plaintext and public-structure cryptanalysis method, the Error Function Attack (EFA), for the analysis of chaos encryption. Since the only thing Eve needs to do is to find out the secret key $k$, the most straightforward approach would be for her to try every possible key, $k^{\prime}$, that Alice and Bob probably can use (the keyspace). This is the so called brute-force attack. By defining the EFA function

$$
e\left(k^{\prime}\right)=\frac{1}{T} \int_{0}^{T}\left|D_{k^{\prime}}(C(t))-P(t)\right| d t=\frac{1}{T} \int_{0}^{T}\left|D_{k^{\prime}}\left(E_{k}(P(t))\right)-P(t)\right| d t,
$$

Eve can scan the whole keyspace to find out the proper key $k^{\prime}=k$ which satisfies $e\left(k^{\prime}\right)=0$. 
Here $T$ represents the amount of data Eve will use, $d t$ represents the length of each block of plaintext $(P(t))$ and ciphertext $(C(t)), E_{k}(P)$ represents the encryption process and $D_{k^{\prime}}(C(t))$ denotes the decryption process.

For public-structure and known-plaintext attack, there are many well developed methods in conventional cryptanalysis, such as the linear attack and the differential attack. These methods have proved to be ineffective when used on chaos encryption systems [8]. As we mentioned in Sec. I, the basic difference between conventional cryptography and chaos cryptography is that the conventional encryption is defined on discrete sets and the chaos encryption is defined on continuous sets. This makes the keyspace behavior of chaotic

systems very different from that of conventional systems [8, 15]. Due to its continuous-value property, in chaos encryption systems keys that are not identical but are very close can still be used to synchronize the two systems very well, thus will form a key basin around the actual secret key [7, 9, 13, 15]. Because of this, Eve will not need to try all the keys in the keyspace; she can, according to the special characters of key basin, find some optimization method to systematically adjust the trial key $k^{\prime}$ untill $k^{\prime}=k$. By this way, the cryptanalysis time can be greatly reduced.

In addition to this, EFA also attacks the weakest part in chaos encryption systems the nonchaotic receiver. Since the dynamics of the receiver is definitely not sensitive to the initial conditions and may not be sensitive to the parameter mismatch as well, it is much easier to attack the receiver than to attack the transmitter whose sensitivity depends on both parameters and initial conditions. Thus, as a public-structure and known-plaintext attack, EFA not only fully exploits the information of the known structure and known plaintext, but also enjoys the advantage of considerable keyspace reduction by attacking the nonchaotic receiver.

\section{EFA ANALYSIS ON DIFFERENT CHAOS ENCRYPTION SCHEMES}

For any encryption scheme, the most important evaluation is about its security. In Sec. II, we had mentioned that EFA has many advantages for cryptanalysis of chaos based encryption. In this section, we will employ this method to evaluate the security of some popular encryption schemes. Specifically, the active-passive decomposition model, the piecewise linear function model, the time delay model, the noise driving sequential synchronization 
model, and the one-way coupled map lattices model. All these models apply chaos synchronization and make use of high dimensional hyperchaos, i.e. they are assumed to have better security than low dimensional chaos. According to the requirement of the Kerckhoffs

principle, we consider only systems with the public-structure, i.e., the entire dynamics is opened to the public except a single parameter which serves as the secret key. Meanwhile, we assume that the eavesdropper could obtain some past plaintext-ciphertext pairs, namely, we consider the public-structure and known-plaintext attack. EFA will be used for analyzing all these cryptosystems.

\section{A. Active-passive decomposition (APD) model}

Proposed in 1995 by Kocarev et al and later generalized widely, the active-passive decomposition (APD) model has been one of the most popular chaos encryption schemes studied during the past years [16]. This model has two important characteristics: (a) the message is not added directly to the chaotic carrier but drives the dynamical system constituting the transmitter; (b) the dimension of encryption system can be arbitrarily high by cascading identical or different individual systems, and thus the APD model had been assumed with high security in early time. For the consideration of the public-structure and knownplaintext attack, each parameter in the equations can be regarded as a secret key in the APD model. Here we use cascaded Rössler systems investigated in Ref. [16] as our model:

Transmitter:

$$
\begin{aligned}
& \dot{x}_{1}=2+x_{1}\left(y_{1}-4\right), \\
& \dot{y}_{1}=-x_{1}-z_{1}, \\
& \dot{z}_{1}=y_{1}+a_{1} s_{1},
\end{aligned}
$$

with

$$
s_{1}=z_{1}+0.25 P
$$

and

$$
\begin{aligned}
& \dot{x}_{i}=2+x_{i}\left(y_{i}-4\right), \\
& \dot{y}_{i}=-x_{i}-z_{i}, \\
& \dot{z}_{i}=y_{i}+a_{i} s_{i},
\end{aligned}
$$


with

$$
s_{i}=z_{i}+0.25 s_{i-1}, \quad i=2,3, \ldots . n .
$$

Here $P$ stands for the message to be transmitted privately and $s_{n}$ represents the output ciphertext. All the parameters $a_{i}, i=1,2, \ldots, n$, can be used as secret keys and are chosen carefully to keep the last oscillator staying within chaos. In order to simplify the related analysis, we set $P(t)=0$ in Eq. (1) for each scheme investigated in this paper without losing generality. In fact, the APD model performs a multiple encryption process: the plaintext is first encrypted to $s_{1}$ by the first Rössler oscillator, then $s_{1}$ is regarded as the new input plaintext and encrypted to $s_{2}$ by the second Rössler oscillator, and the same process repeated down to the last Rössler oscillator which generates $s_{n}$ as the last output ciphertext. On the receiver side, the whole system is identical to the transmitter except the encryption process be reversed,

Receiver:

$$
\begin{aligned}
& \dot{x}_{n}^{\prime}=2+x_{n}^{\prime}\left(y_{n}^{\prime}-4\right), \\
& \dot{y}_{n}^{\prime}=-x_{n}^{\prime}-z_{n}^{\prime}, \\
& \dot{z}_{n}^{\prime}=y_{n}^{\prime}+a_{n}^{\prime} s_{n}^{\prime},
\end{aligned}
$$

with

$$
s_{n}^{\prime}=s_{n}
$$

and

$$
\begin{aligned}
& \dot{x}_{i}^{\prime}=2+x_{i}^{\prime}\left(y_{i}^{\prime}-4\right), \\
& \dot{y}_{i}^{\prime}=-x_{i}^{\prime}-z_{i}^{\prime}, \\
& \dot{z}_{i}^{\prime}=y_{i}^{\prime}+a_{i}^{\prime} s_{i}^{\prime},
\end{aligned}
$$

with

$$
s_{i}^{\prime}=\left(s_{i+1}^{\prime}-z_{i+1}^{\prime}\right) / 0.25, \quad i=n-1, n-2 \ldots . .1 .
$$

By setting $a_{i}^{\prime}=a_{i}$, the receiver can be synchronized with the transmitter and the message can be recovered step by step using Eq. (9), and the original message will be recovered at the last oscillator by using $P=\left(s_{1}^{\prime}-z_{1}^{\prime}\right) / 0.25$. 
Now we use EFA to analyze the key behavior of this encryption model. Setting $a_{j}=$ $0.45, j=1,2, \ldots n$, the transmitter is spatiotemporally chaotic with $n$ positive LEs. In our simulations we choose the most effective (For the APD model, systems are cascaded unidirectionally. The more last the parameter is, the more sensitive the decryption process will be, this is also verified by our numerical simulations.) parameter $a_{n}$ the parameter of the last Rössler oscillator, as the secret key for encryptions and define the keyspace by keys that keep the last Rössler oscillator in the chaotic regime. For Eve, she has all the knowledge about the transmitter and the receiver except $a_{n}$, and she also knows the range of the keyspace. Consequently, she can run the receiver, Eqs. (6)-(9), by choosing the trial key $a_{n}^{\prime}$ randomly within the keyspace, and calculate the corresponding error function results by using Eq. (1). In Fig. 1(a), we plot the key basin for the $n=2$ APD model by using EFA

$$
e\left(a_{2}^{\prime}\right)=\frac{1}{T} \int_{0}^{T}\left|z_{1}^{\prime}\left(a_{2}^{\prime}\right)-z_{1}\left(a_{2}\right)\right| d t .
$$

It is found that the whole keyspace $a_{2}^{\prime} \in[0.44,0.46]$ forms a single key basin, two straight lines with reversed slopes dominate the behavior of $e\left(a_{2}^{\prime}\right)$. The secret key $a_{2}^{\prime}=a_{2}=0.45$ is located at the minimum point $e\left(a_{2}^{\prime}=a_{2}\right)=0$. With this structure, Eve can find the correct key easily through some adaptive adjustments. For example, Eve can first try arbitrarily two trial keys in the keyspace, $a_{2}^{\prime}(1)$ and $a_{2}^{\prime}(2)$, and by comparing the respective values of $e$, she will know which direction she should be adjusting her next attempt. In our simulations, only 6 to 8 tests are needed to find the properly location of $a_{2}$. Furthermore, by using the slopes, we can evaluate $a_{2}$ proximately only by two trial keys. We refer to this kind of key basin as the triangle basin and the method for key searching mentioned above as the adaptive adjustment method (AAM). It is obvious that this model has no security against EFA.

In order to investigate the relationship between the dimension and the security in this type of scheme, we also plot the key basins for $n=3,4,5$ in Fig. 1(b). As the dimension of the system increases, the key basin changes only in shape, but the structure of triangle basin still persists. This kind of basin structure can also be found in other APD based models, and in Fig. 2 we plot the related key basins for the model used in Ref. [17]. All parameters and dynamics of the systems are those of the original paper and the parameter in the equation of the first variable is chosen as the key. We find that no matter how high the 
system dimension is (the dimension changes from 5 to 101 in our simulations), the triangle basin remains. (We should mention that as the system dimension is increased, we also see a longer transient time before the triangle basin becomes manifest.) Therefore, increasing the system size (i.e., the dimension of hyperchaos) does not lead to an increase in security for this encryption scheme. This result is rather surprising, and this behavior should be seriously considered whenever one hopes to reach high security by increasing the dimension of chaotic system, or say, by applying spatiotemporal chaos.

\section{B. Coupled piecewise linear function (CPLF) model}

For a long time, the piecewise linear function model has been another popular nonlinear model investigated extensively [18, 19, 20]. There exists a well-developed theory of piecewise linear maps which generate uniformly distributed signals, and it is known that piecewise linear maps share nice properties of invariant measures, ergodicity and statistical independence [13]. In Ref. [19], the authors proposed an efficient encryption scheme based on coupled piecewise linear maps, and they declared that such cryptosystems not only enjoy high security, but also have an "immense parameter space" for key choosing even in lower dimensional encryption systems. Here we choose the five-dimensional system used there as our model and analyze its security by using EFA. The dynamics of the transmitter and the receiver can be written as:

Transmitter:

$$
\begin{aligned}
x_{5}(k-1)= & f_{1}\left[x_{3}(k-1)\right]+a_{51} x_{1}(k-1)+a_{52} x_{2}(k-1)+a_{53} x_{3}(k-1) \\
& +a_{54} x_{4}(k-1)+k_{7} x_{2}(k-1) \sin \left[k_{8} x_{3}(k-1)\right], \\
x_{1}(k)= & f_{2}\left[x_{5}(k-1)\right]+c \cdot P(k), \\
x_{2}(k)= & f_{3}\left[x_{3}(k-1)+x_{4}(k-1)\right]+x_{1}(k)+a_{22} x_{2}(k-1)+a_{23} x_{3}(k-1) \\
& +a_{24} x_{4}(k-1)+k_{1} x_{2}(k-1) \sin \left[k_{2} x_{3}(k-1)\right], \\
x_{3}(k)= & f_{4}\left[x_{4}(k-1)\right]+a_{32} x_{2}(k-1)+a_{33} x_{3}(k-1)+a_{34} x_{4}(k-1) \\
& +k_{3} x_{3}(k-1) \sin \left[k_{4} x_{4}(k-1)\right], \\
x_{4}(k)= & a_{42} x_{2}(k-1)+a_{44} x_{4}(k-1)+k_{5} x_{4}(k-1) \sin \left[k_{6} x_{2}(k-1)\right],
\end{aligned}
$$


with the piecewise linear map

$$
f_{i}(x)=C_{0}+C_{1} x+\sum_{j=1}^{N} D_{j} \cdot\left|x-E_{j}\right|, \bmod \chi .
$$

Receiver:

$$
\begin{aligned}
x_{4}^{\prime}(k)= & a_{42}^{\prime} x_{2}(k-1)+a_{44}^{\prime} x_{4}^{\prime}(k-1)+k_{5}^{\prime} x_{4}^{\prime}(k-1) \sin \left[k_{6}^{\prime} x_{2}(k-1)\right], \\
x_{3}^{\prime}(k)= & f_{4}^{\prime}\left[x_{4}^{\prime}(k-1)\right]+a_{32}^{\prime} x_{2}(k-1)+a_{33}^{\prime} x_{3}^{\prime}(k-1)+a_{34}^{\prime} x_{4}^{\prime}(k-1) \\
& +k_{3}^{\prime} x_{3}^{\prime}(k-1) \sin \left[k_{4}^{\prime} x_{4}^{\prime}(k-1)\right], \\
x_{1}^{\prime}(k)= & x_{2}(k)-f_{3}^{\prime}\left[x_{3}^{\prime}(k-1)+x_{4}^{\prime}(k-1)\right]-a_{22}^{\prime} x_{2}(k-1) \\
& -a_{23}^{\prime} x_{3}^{\prime}(k-1)-a_{24}^{\prime} x_{4}^{\prime}(k-1)-k_{1}^{\prime} x_{2}(k-1) \sin \left[k_{2}^{\prime} x_{3}^{\prime}(k-1)\right], \\
x_{5}^{\prime}(k-1)= & f_{1}^{\prime}\left[x_{3}^{\prime}(k-1)\right]+a_{51}^{\prime} x_{1}^{\prime}(k-1)+a_{52}^{\prime} x_{2}(k-1) \\
& +a_{53}^{\prime} x_{3}^{\prime}(k-1)+a_{54}^{\prime} x_{4}^{\prime}(k-1)+k_{7}^{\prime} x_{2}(k-1) \sin \left[k_{8}^{\prime} x_{3}^{\prime}(k-1)\right], \\
P^{\prime}(k)= & \left\{x_{1}^{\prime}-f_{2}^{\prime}\left[x_{5}^{\prime}(k-1)\right]\right\} / c .
\end{aligned}
$$

This is a delicately designed cascaded system where the plaintext $P$ is added to the first variable and influences all the other variables through couplings. The variable $x_{2}$ serves as the ciphertext and also acts as the driver signal of the receiver for chaos synchronization. In the piecewise linear map $f_{i}(x), E_{1}<E_{2}<\ldots E_{N}$ are breaking points and $C_{1}=\frac{1}{2}\left(m_{0}+m_{N}\right)$, $D_{j}=\frac{1}{2}\left(m_{j}-m_{j-1}\right), C_{0}$ are parameters used for model adjustment, and $m_{j}$ is the slope for the $j$ th segment. In our simulations, we set $N=20, \chi=100$ and set $E_{j}$ uniformly distributed in $[0, \chi]$ so as to simplifying our analysis. We also set the slope $m_{j}=N \times \frac{\sqrt{5}-1}{2}>1$ so that the system stays within the chaotic regime. With $C_{0}=-50$, the function of one single piecewise linear map is plotted in Fig. 3(a).

In this cryptosystem, all coupling parameters and parameters in each map function can be used as secret keys. Here we use the most sensitive parameter $a_{44}$ as the secret key and keep all the other parameters public. According to the requirement of convergence we fix the whole parameter set as

$$
\begin{aligned}
a_{22} & =0.1, a_{23}=-0.09, a_{24}=0.1, a_{32}=0.1, a_{33}=0.1, a_{34}=0.2, \\
a_{42} & =-0.23, a_{51}=0.1, a_{52}=0.3, a_{53}=0.1, a_{54}=0.31, \\
c_{1} & =0.06, c_{2}=3.4, c_{3}=0, c_{4}=12.9, c_{5}=0.378, c_{6}=-0.99 \\
c_{7} & =0.001, c_{8}=1 .
\end{aligned}
$$


For $a_{44}=-0.89$, we plot in Fig. 3(b) the key basin for $a_{44}^{\prime}$ by using the EFA

$$
e\left(k^{\prime}\right)=\frac{1}{T} \sum_{k=1}^{T}\left|P(k)-P^{\prime}\left(k^{\prime}\right)\right| .
$$

It is found that there exists a key basin $a_{44}^{\prime} \in[-0.895,-0.885]$ which occupies a parameter interval of order $10^{-2}$ around the actual secret key. Compared with the entire keyspace $a_{44}^{\prime} \in[-1,-0.75]$, which is of the order of $10^{-1}$, it is still an relatively easy job to expose the secret key. For example, in order to attack this system, Eve can divide the whole keyspace into some key basin sized intervals, then test a few trial keys in each intervals to find where the actual key basin is located. Once this interval is identified, Eve can the focus her searching in this basin and determine the secret key by AAM as used for the APD model. For the above mentioned five-dimensional model, we can extract the secret key within 200 tests, a considerably reduced searching time in comparison to the brute-force attack which needs about $10^{15}$ tests [19].

Although still not quite secure enough, the security of the CPLF model has been improved greatly compared to the APD model. Moreover, the security of CPLF can be considerably improved by increasing the number of modulo operations. With all other parameters unchanged, we plot in Fig. 3(c) and (d) the EFA results for $C_{0}=0$ (the function of one single piecewise linear map shown in Fig. 3(a) is divided into two separate parts this time) and $m_{j}=10 \times N \times \frac{\sqrt{5}-1}{2}$, respectively - both settings increase the number of modulo operations. It is apparent that the system security is significant improved.

\section{Delay-differential equations (DDE)}

The time delay system employing delay-differential equations is an efficient model for constructing high dimensional hyperchaos. Its dynamics structure is rather simple, but its sequences can be very complex 21]. Besides, DDE can be easily implemented in electronic

systems 22]. Comparing with other hyperchaotic systems, DDE has some special advantages for encryption. First of all, DDE is an infinite dimensional dynamics with a high dimensional attractor with many positive LEs; these guarantee high complexity of its output signals. Secondly, the complexity and the number of positive LEs can be controlled easily by adjusting the delay time $\tau$. In these systems, we do not need to worry about the problem of weak keys, since the system will always be chaotic and the number of positive 
LEs will increase linearly, once $\tau$ exceeds the critical value. Finally, synchronization between DDE has been established and proved to be robust [23]. Therefore, DDE has been a popular model for encryption in both theoretical and experimental investigations [9, 24, 25].

For this kind of cryptosystems, the delay time $\tau$ is the most suitable choice for the secret key. Here we employ the Mackey-Glass DDE (MG DDE) cryptosystem used in Ref. [26] as our model and analyze its security by EFA. The dynamics of the transmitter and the receiver are

Transmitter:

$$
\frac{d x(t)}{d t}=-b x(t)+\frac{a x(t-\tau)}{1+x^{c}(t-\tau)}+\epsilon P(t)
$$

receiver:

$$
\frac{d y(t)}{d t}=-b y(t)+\frac{a y\left(t-\tau^{\prime}\right)}{1+y^{c}\left(t-\tau^{\prime}\right)}+[s(t)-y(t)]
$$

with

$$
s(t)=x(t)+\epsilon P(t) .
$$

It was shown that with parameters $b=0.1, a=0.2$, and $c=10$, the above system will always be kept in the chaotic regime for $\tau>16.8$. For $\tau=300$, there are altogether 15 positive LEs and the system dimension is about 30. As $\tau$ increases, both the number of positive LEs and the Kaplan-Yorke dimension increase [21]. In our simulations, we consider the keyspace with the range $\tau^{\prime} \in[150,450]$ and choose $\tau=300$ as the actual secret key. The key basin is plotted in Fig. 4 using the EFA

$$
e\left(\tau^{\prime}\right)=\frac{1}{T} \int_{0}^{T}\left|x_{\tau}(t)-y_{\tau^{\prime}}(t)\right| d t
$$

In this case, the key basin is the range $\tau^{\prime} \in[230,340]$, and considering the $\mathrm{O}\left(10^{2}\right)$ size of the entire keyspace, it is obviously an easy job to determine the secret key by AAM as in the cases discussed above.

\section{Noise driven sequential synchronization (NDSS) model}

A hierarchically structured cryptosystem is proposed recently [27], employing sequentially synchronized chaotic systems. Sequential synchronization is attained by first feeding a noiselike signal to a variable of the first transmitter and its receiver simultaneously and then feeding a variable of the first transmitter and its receiver to a variable of the second 
transmitter and its receiver, respectively, and repeating the feedings of successive variables in sequence. Plaintext is added directly to the variables to form the ciphertext on the transmitter side, and is recovered by synchronization on the receiver side. This is different from the encryption schemes mentioned above, as the plaintext here is not involved in the dynamics. Such an encryption scheme appears to have high security, which can be enforced selectively: different users can maintain different security levels according to the synchronization level that can be reached. Here we consider the cryptosystem composed of one Navier-Stokes oscillator and one Lorenz oscillator, as used in Ref. [27], with both the transmitter and the receiver sharing the same dynamics,

$$
\begin{aligned}
& \dot{x}=-1.9 x+4\left[\alpha_{1} y+\beta_{1} f(t)\right] z+4 u v, \\
& \dot{y}=-7.2\left[\alpha_{1} y+\beta_{1} f(t)\right]+3.2 x z, \\
& \dot{z}=-4.7 z-7.0 x\left[\alpha_{1} y+\beta_{1} f(t)\right]+k \\
& \dot{u}=-5.3 u-x v \\
& \dot{v}=-v-3.0 x u, \quad \text { (Navier-Stokes) } \\
& \dot{p}=\sigma\left[\left(\alpha_{2} q+\beta_{2} z\right)-p\right] \\
& \dot{q}=c p-\left(\alpha_{2} q+\beta_{2} z\right)-p r \\
& \dot{r}=p\left(\alpha_{2} q+\beta_{2} z\right)-b r, \quad \text { (Lorenz) }
\end{aligned}
$$

where $\alpha_{1}, \alpha_{2}, \beta_{1}$ and $\beta_{2}$ are the couplings, $f(t)$ is the noise signal which reads

$$
f(t)=50 \xi \sin \left[2 \pi \times 0.8\left(\frac{1}{2}+\xi^{\prime}\right) t\right]
$$

where $\xi$ and $\xi^{\prime}$ are pseudorandom numbers within $(0,1)$.

With the parameters $k, \sigma, c$, and $b$ taken to be $36,10.0,28.0$, and $8 / 3$, respectively, for $\alpha_{1}=1.2, \beta_{1}=0.9, \alpha_{2}=0.9$, and $\beta_{2}=22.5$, both the transmitter and the receiver Exhibit chaotic behavior but can be synchronized. For public-structure and known-plaintext attack, we choose the parameter $k=36$ in the Navier-Stokes equations as the secret key and consider the keyspace $k^{\prime} \in[35,37]$, where the whole system stays in the chaotic regime and synchronization between the transmitter and the receiver can be achieved. In our simulations, we choose the variables $v$ and $r$ as the carriers, and use the EFA

$$
e\left(k^{\prime}\right)=\frac{1}{T} \int_{0}^{T}\left|v^{\prime}\left(k^{\prime}\right)-v(k)\right| d t
$$


and

$$
e\left(k^{\prime}\right)=\frac{1}{T} \int_{0}^{T}\left|r^{\prime}\left(k^{\prime}\right)-r(k)\right| d t .
$$

The key basins are plotted for the Navier-Stokes system and the Lorenz system in Fig. 5(a) and Fig. 5(b), respectively. Again we find the triangle basin in the Navier-Stokes system, with a similar one for the Lorenz system, except with a little distortion. (This appears to be typical for every variable in this system chosen as the secret key.) The conclusion is clear: the secret key can be easily determined using AAM just as in the cases that we have discussed, and the claim for high security does not seem to be justified. From the results of our simulations, we do not see any improvement with more complicated coupled chaotic systems.

\section{E. One-way coupled map lattices (OCML)}

For a long time, coupled map lattices (CML) have been used to investigate the complex behavior of spatiotemporal chaos in many fields of nonlinear science [28]. Recently, this kind of system has been utilized for secure communication in a number of encryption algorithms. In particular, the one-way coupled map lattices (OCML) is extensively used for self-synchronizing, spatiotemporal chaos-based cryptosystems [29, 30].

The earlier works on OCML inherited the classical ideas of chaos encryption: they regarded OCML as a special spatiotemporal chaos system with inherent high computational complexity and yet amendable to easy analysis [29]. Later, modified OCML models were proposed to make the systems more feasible for encryption application. These modifications include the adoption of self-synchronization, the use of binary sequences more suitable for digital communications [12], and the application of modulo operations to improve the system security [30], etc. Further studies show that OCML ciphers can be improved to have not only competitive security when compared against conventional ciphers, but also respectable encryption speed and low bit error [30]. In the following, we shall mainly evaluate the property of security in a OCML system by employing the EFA.

We use a modified two-dimensional OCML encryption system recently proposed in Ref. 31] as our model. The transmitter is comprised of two parts: the first part is a one- 
dimensional OCML with $m$ coupled lattices,

$$
\begin{aligned}
x_{0}(n) & =S_{N / 2, N / 2}(n) / 2^{v}, \\
x_{l}(n+1) & =(1-\varepsilon) f_{l}\left[x_{j}(n)\right]+\varepsilon f_{l-1}\left[x_{l-1}(n)\right], \\
f_{l}(x) & =a_{l} x(1-x), \quad l=1,2, \ldots m,
\end{aligned}
$$

while the second part is a two-dimensional OCML driven by the first part through lattice $z$,

$$
\begin{aligned}
z(n+1) & =(1-\varepsilon) f[z(n)]+\varepsilon f_{m}\left[x_{m}(n)\right], \\
y_{0,0}(n) & =z(n) \times 2^{h} \bmod 1, \\
y_{1,0}(n+1) & =(1-\varepsilon) f\left[y_{1,0}(n)\right]+\varepsilon f\left[y_{0,0}(n)\right], \\
y_{0,1}(n+1) & =(1-\varepsilon) f\left[y_{0,1}(n)\right]+\varepsilon f\left[y_{0,0}(n)\right], \\
y_{i, 0}(n+1) & =(1-\varepsilon) f\left[y_{i, 0}(n)\right]+\varepsilon\left\{0.8 f\left[y_{i-1,0}(n)\right]+0.2 f\left[y_{i-2,0}(n)\right]\right\}, i=2, . . N, \\
y_{0, j}(n+1) & =(1-\varepsilon) f\left[y_{0, j}(n)\right]+\varepsilon\left\{0.2 f\left[y_{0, j-1}(n)\right]+0.8 f\left[y_{0, j-2}(n)\right]\right\}, j=2, . . N, \\
y_{i, j}(n+1) & =(1-\varepsilon) f\left[y_{i, j}(n)\right]+\varepsilon\left\{0.5 f\left[y_{i-1, j}(n)\right]+0.5 f\left[y_{i, j-1}(n)\right]\right\}, 2 \leq i+j \leq N, \\
f(x) & =4 x(1-x) .
\end{aligned}
$$

The output ciphertext reads

$$
\begin{aligned}
& S_{i, j}(n)=\left[K_{i, j}(n)+P_{i, j}(n)\right] \quad \bmod 2^{v}, \\
& K_{i, j}(n)=\left\{\operatorname{int}\left[y_{i, j}(n) \times 2^{\eta}\right]\right\} \quad \bmod 2^{v}, 2 \leq i+j \leq N .
\end{aligned}
$$

The dynamics of the receiver is identical to that of the transmitter. When the two systems are synchronized, messages can be recovered by the reverse process of Eqs. (26). The detailed explanation on the structure of Eqs. (25) and Eqs. (26) is found in Ref. [31], and in this work we shall focus on analyzing its security and other encryption properties. For this cryptosystem, the parameters $a_{l}, l=2,3, \ldots m$, can be used as the secret keys. In the following simulations, we set the parameters $N=6, m=3, \varepsilon=0.99, h=26, \eta=60$, $v=30, a_{l}=3.9, l=2,3, \ldots m$, and choose just one parameter (just as we did for the previous four models), $a_{1}$, as the secret key. Except for the lattice $y_{0,0}$, each lattice in the second part can be used as an encryption unit, thus significantly enhancing the encryption speed. (With the above settings, for example, the system can generate a total of 25 sequences simultaneously.) Moreover, the modulo operations used in Eqs. (25) and Eqs. (26) also 
serve to greatly improve the system security. This is therefore a system that potentially has both high security and fast encryption speed.

With the requirement of public-structure and known-plaintext, we study system's key basin by using the EFA

$$
e_{i, j}\left(a_{1}^{\prime}\right)=\frac{1}{T} \sum_{n=1}^{T}\left|K_{i, j, a_{1}}(n)-K_{i, j, a_{1}^{\prime}}^{\prime}(n)\right|,
$$

with $K_{i, j, a_{1}^{\prime}}^{\prime}$ representing the corresponding output in the receiver for the trial key $a_{1}^{\prime}$. In Fig. 6 we plot the simulation results for $e_{3,3}$ with the secret key chosen as $a_{1}=3.9$. The key basin is observed to be within the range $\left[3.9-4 \times 10^{-12}, 3.9+4 \times 10^{-12}\right]$. Since the whole keyspace

for this kind of system is at least within range $a_{1}^{\prime} \in[3.6,4.0]$, it needs at least $10^{11}$ tests to determine the correct secret key using EFA. We have also investigated through simulations the key basins for other output sequences $S_{i, j}$, and found that the widths of the key basins typically range from $10^{-11}$ to $10^{-12}$, depending on where the output lattice is located. This suggests that any one output sequence is a good candidate for encryption. In comparison with other four models mentioned above, this model appears to possess much higher system security.

\section{QUALITY FACTOR}

In conventional cryptography, three criteria are commonly considered when designing an effective and applicable cryptosystem: security, the encryption speed, and the error rate 14]. For chaos based encryption, relatively little attention has been paid to the latter two criteria [1, 13, 15], which are intimately linked to the attempts to achieve greater security. First of all, security of a cryptosystem depends mainly on its dimension: the higher the dimension, the more complex the signal it can generate. However, high dimensional systems usually incur greater encryption time cost in both software and hardware implementations. Obviously there will have to be a trade-off between these two in considering the realistic application of a system. Furthermore, higher system security usually implies higher system sensitivity, and this brings about another problem for chaos encryption, name the system stability. Both the encryption systems and the communication channels can be disturbed unavoidably for various reasons like intentional attacks or unintentional perturbations). The system sensitivity of a high security system will amplify these disturbances rapidly. This 
is particularly problematic for chaos synchronization based cryptosystems which have to spend a certain time to eliminate these disturbances before any correct communication can be accomplished. All these highlight the relevance of considerations beyond just system security in evaluating the overall performance and applicability of any given encryption scheme. In this section, we will analyze the encryption models used in Sec. III by studying their performance in all the three aspects mentioned above, and compare their advantages and drawbacks from the point of application.

Before going further, we need to give the security a quantitative description. Based on the analysis of EFA, there always exists a key basin around the actual secret key. Without the knowledge of this basin, one may not be able to extract the secret key by brute-force or other means of searching. We define the key basin width, denoted by $w$, by the distance between two trial keys located on the two sides of the key basin, which exceeds the average EFA result of the entire keyspace (see the marked regions in Fig. 3(b), Fig.4, and Fig. 6). Here, we call the number of intervals (which are potential key basins) with this width in the whole keyspace as the key number. (For different choices of the secret key, there can be small differences in the basin widths.) The security of the system is broken as soon as the actual key basin is exposed, since the searching cost of extracting the secret key then once is negligible. This motivates us to define the security of a cryptosystem as the entropy of the key number [15]

$$
S=\log _{2} \frac{K}{w},
$$

with $K$ denoting the length of the entire keyspace and $\frac{K}{w}$ being the key number.

We define the amount (megabit) of data that can be encrypted in each second as the encryption speed, denote it by $V$. The number $L$ of bits that are affected when one bit is in error in the ciphertext is defined as the error propagation length (EPL). Our simulation study is based on software implementation, and treat each variable as a 8-byte (i.e. 64-bit, double precision, real) data. In computing the EPL, random perturbation is added within the range of the output signals, and the average over 1000 runs is taken.

As we had emphasized above, there exist some trade-off relations between security, encryption speed and EPL, so from the point of application, we need to strike a balance between these aspects so as to obtain a better overall performance. In this paper, we represent this 
balance by the Quality Factor, which we define as

$$
B=\frac{V S}{L} \text {. }
$$

Although this definition may not reflect the precise relationship in real applications, the Quality Factor does parameterize the interplay of the factors affecting the overall performance of a cryptosystem in a concrete and reasonable way. but it can fairly well reflect some basic properties for evaluating system's overall performance. We find the following results for the different encryption models discussed in Sec. III:

\begin{tabular}{|c|c|c|c|c|c|}
\hline & APD & CPLF & DDE & NDSS & OCML \\
\hline$S$ & $\log _{2} 1$ & $\log _{2} 10^{3}$ & $\log _{2} 10^{1}$ & $\log _{2} 1$ & $\log _{2} 10^{11}$ \\
\hline$V$ & 31.6 & 2.8 & 24.5 & 22.6 & 216.7 \\
\hline$L$ & 1737 & 681 & 719 & 2213 & 22 \\
\hline$B$ & 0 & 0.041 & 0.113 & 0 & 360 \\
\hline
\end{tabular}

The above results are obtained on SGI OCTANE workstation (two 195MHZ IP30 CPU 256M RAM, Fortran90 compiler). We have also carried out computations on other workstations and compilers and obtained qualitatively similar results. For the simulations, we choose the parameters as for Fig. 1(a) for the APD model, Fig. 3(a) for the CPLF model, Fig. 4 for the DDE model, Figs. 5(a) and (b) for the NDSS model, and Fig. 6 for the OCML model, respectively, so as to maximize the overall performance for each scheme.

Based on the above table, we wish to make the following observations.

1. From the security point of view, the OCML and CPLF models perform much better than the other models. This is mainly due to the modulo operations employed in these two schemes. The modulo operation disposes the most significant digits in the signals and keep only those less significant digits, making the systems more sensitive to the key values. As verification, we have also tried applying modulo operations on the output signals of the NDSS model (the same parameters as in Figs. 5(a) and (b)), and the related EFA results are plotted in Fig. 7(a) and (b) for the Navier-Stokes system and the Lorenz system, respectively. The improvement in the system security is obvious.

2. The OCML model possesses excellent encryption speed due to its simple functions and multiple outputs. In our simulation, we find that although the modulo operation can 
improve appreciably the system security greatly, it is also a time-consuming function. This is largely responsible for the low encryption speed of the CPLF model. In contrast, the 25 simultaneous outputs and the simplicity of the mapping functions more than compensate for the extra processing time linked to the modulo operations.

3. Finally, from the results on the EPL, it is clear that lower dimensional, mapping systems perform better than high-dimensional, differential systems. Moreover, systems with multiple outputs but with only one as the driving signal has a clear advantage in EPL performance. While the errors in the transmitted bits of the driving signal are responsible for the EPL, the remaining nondriving ciphertext bits do not give rise to such a problem, i.e., one bit error of the transmitted ciphertext causes only one bit error in the received plaintext. Together with the two dimensional structure design and strong couplings, these features give the OCML model an outstanding performance as far as EFL is concerned.

\section{CONCLUSION}

In this paper, we proposed an efficient cryptanalysis tool, the EFA method, to study the security of some well-known chaos encryption schemes. Our systematic comparison suggests that even models (such as the APD and NDSS models) with high dimensionalities (and hence the supposed higher security) fail to maintain their security under the EFA. We are also of the opinion that in addition to security consideration, there are other important aspects of cryptosystems which affect their overall Performance. More specifically, we consider, additionally, the encryption speed and the error propagation Length, and suggest in this paper a quantity (the Quality Factor) as a possible measure of the overall performance, or applicability, of chaos-based cryptosystems. Through comparisons, we find that the modified OCML model has the best overall performance among the models considered, and some the reasons responsible for this performance are briefly discussed. 
[1] L. Kocarev, IEEE Circuits syst magz., 1(3), 6 (2001).

[2] C.E. Shannon, Bell Syst. Tech. J. 28, 656 (1949).

[3] R. Matthews, Cryptologia, XIII(1), 29 (1989); D.D. Wheeler, Cryptologia, XIII(3), 243 (1989); D.W. Mitchell, Cryptologia XIV(4), 350 (1990); E. Biham, Advances in CryptologyCRYPTO'94, Y.G. Desmedt, Ed. New York: Springer-Verlag, 839, 318 (1994).

[4] S. Hayes, C. Grebogi, E. Ott, A. Mark, Phys. Rev. Lett. 73, 1781 (1994); D. Gligoroski, D. Dimovski, L. Kocarev, V. Urumov, L.O. Chua, Int. J. Bif Chaos Appl. Sci. Eng. 6, 2119 (1996); M.S. Baptista, Phys. Lett. A 240, 50 (1998).

[5] L.M. Pecora, T.L. Carroll, Phys. Rev. Lett. 64821 (1990); T.L. Carroll and L.M. Pecora, IEEE Trans. Circuits Syst. 38, 453 (1991).

[6] L.M. Cuomo, A.V. Oppenheim, phys. Rev. Lett. 71, 65 (1993).

[7] G.D. Vanwiggeren and R. Roy, Science 279, 1198 (1998); Phys. Rev. Lett. 81, 3547 (1998); J. Garcia-Ojalvo and R. Roy, Phys. Rev. Lett. 86, 5204 (2001).

[8] L. Kocarev, et al., Int. J. Bif Chaos Appl. Sci. Eng. 2, 709 (1992); U. Parlitz, et al., Int. J. Bif Chaos Appl. Sci. Eng. 2, 973 (1992).

[9] C. Zhou, and C.-H. Lai, Phys. Rev. E 60, 320 (1999); V.I. Ponomarenko and M.D. Prokhorov, Phys. Rev. E 66, 26215 (2002).

[10] G. Perez and H.A. Cerdeira, Phys. Rev. Lett. 74, 1970 (1995).

[11] K.M. Short, Int. J. Bif and Chaos. 4(4), 959 (1994); 6(2), 367 (1996); K.M. Short, A.T. Parker, Phys. Rev. E 58, 1159 (1998).

[12] X. Yongxiang, S. Xiuming, R. Yong, Y. Xunhe, and L. Feng, Phys. Rev. E 64, 67201 (2001).

[13] F. Dachselt and W. Schwarz, IEEE Trans. Circuits Syst. 48, 1498 (2001).

[14] B. Scheneier, Applied Cryptography: Protocols, Algorithms, and Source Code in C, Wiley, New York (1996).

[15] B. Fraser, P. Yu, and T. Lookman, Phys. Rev. E 6617202 (2002).

[16] L. Kocarev, U. Parlitz, Phys. Rev. Lett. 74, 5028 (1995); U. Parlitz, L. Kocarev, T. Stojanovski, and H. Preckel, Phys. Rev. E 53, 4351 (1996); L. Kocarev, U. Parlitz, T. Stojanovski, Phys. Lett. A 217, 280 (1996).

[17] G. Baier and S. Sahle, Phys. Rev. E 51, 2712 (1995); U. Parlitz, L. Kocarev, Int. J. Bif Chaos 
Appl. Sci. Eng. 7, 407 (1997).

[18] L.O. Chua, C.A. Desoer, and E.S. Kuh, Linear and Nonlinear Circuits. McGraw-Hill (1987).

[19] S. Papadimitriou, A. Bezerianos, and Tassos Bountis, IEEE Transaction on Computers. 48, 27 (1997); S. Papadimitriou, G. Pavlides, A. Bezerianos, and T. Bountis, Proc. 1995 IEEE Workshop on Nonlinear Signal and Image Processing, 20, 145 (1995).

[20] A.M. Batista, S.E. de S. Pinto, R.L. Viana, and S.R. Lopes, Phys. Rev. E 65, 56209 (2002); V. Varadan and H. Leung, IEEE Trans. Circuits Syst. 49, 1543 (2002).

[21] J.D. Farmer, Physica D 4, 366 (1982); S. Lepri, G. Giacomelli, A. Politi, F.T. Arecchi, Physica D 70, 235 (1993); D.E. Sigeti, Physica D 82, 136 (1995); B. Mensour, A. Longtin, Physica D 113,1 (1998).

[22] J. Losson, M.C. Mackey, A. Longtin, Chaos 167 (1993); A. Namajunas, K. Pyragas, A. Tamasevicius, Phys. Lett. A 201, 42 (1995).

[23] M. J. Bunner and W. Just, Phys. Rev. E 58, 4072 (1998); S. Boccaletti, D.L. Valladares, J. Kurths, D. Maza, and H. Mancini, Phys. Rev. E 61, 3712 (2000).

[24] B. Mensour, A. Longtin, Phys. Lett. A 244, 59 (1998).

[25] L. Yaowen, G. Guangming, Z. Hong, and W. Yinghai, Phys. Rev. E 62, 7898 (2000).

[26] M.C. Mackey, L. Glass, Science 197, 287 (1977).

[27] C.M. Kim, S. Rim, and W.H. Kye, Phys. Rev. Lett. 88, 14103 (2001).

[28] F.H. Willeboordse, Chaos 2, 423 (1992); F.H. Willeboordse, Phys. Rev. E 47, 1419 (1993);

F.H. Willeboordse, and K. Kaneko, Phys. Rev. Lett. 73, 533 (1994).

[29] J.H. Xiao, G. Hu, and Z. Qu, Phys. Rev. Lett. 77, 4162 (1996); G. Hu, J.H. Xiao, J.Z. Yang, F. Xie and Z. Qu, Phys. Rev. E 56, 2738 (1997).

[30] S.H. Wang, J. Kuang, J. Li, Y. Luo, H. Lu, and G. Hu, Phys. Rev. E 66, 65202 (2002).

[31] G. Hu, et al., (unpublished). 


\section{Figure Captions:}

Fig. 1 (a) Key basin of $a_{2}^{\prime}$ for the APD model composed of two coupled Rössler oscillators, $a_{2}=0.45$ is chosen as the secret key. (b) Key basin of $a_{3}^{\prime}, a_{4}^{\prime}$, and $a_{5}^{\prime}$ for APD models with 3, 4, and 5 coupled Rössler oscillators, respectively.

Fig. 2 Key basins for the model used in Ref. [17].

Fig. 3 The coupling $a_{44}$ in Eqs. (11) is chosen as the secret key. (a) Map of CPLF for $C_{0}=-50$ and $m_{j}=20 \times \frac{\sqrt{5}-1}{2}, j=1,2, \ldots, 20$. (b) Key basin of CPLF model with the same parameters as for (a). (c) Key basin for $C_{0}=0$, and, (d) key basin for $m_{j}=10 \times 20 \times \frac{\sqrt{5}-1}{2}$, $j=1,2, \ldots, 20$, other parameters being the same as for (a).

Fig. 4 Key basin for the DDE model, with $b=0.1, a=0.2$, and $c=10$. For The secret key $\tau=300$, there are 15 positive LEs and system dimension is about 30 .

Fig. 5 The secret key $k=36$ is chosen here, and the difference between the trial key $k^{\prime}$ and the secret key $k, \Delta k=k^{\prime}-k$, is used as variable for the horizontal axis. (a) The key basin for the Navier-Stokes system, (b)The key basin for the Lorenz system.

Fig. 6 The key basin for the OCML model with the chosen secret key $a_{1}=3.9$. The variable for the horizontal axis is the difference between the trial key $a_{1}^{\prime}$ and the actual secret key $a_{1}, \Delta a=a_{1}^{\prime}-a_{1}$.

Fig. 7 The key basins after the introduction of modulo operations for (a) the NavierStokes system and (b) the Lorenz system, respectively. The parameters are the same as for Fig. 5 

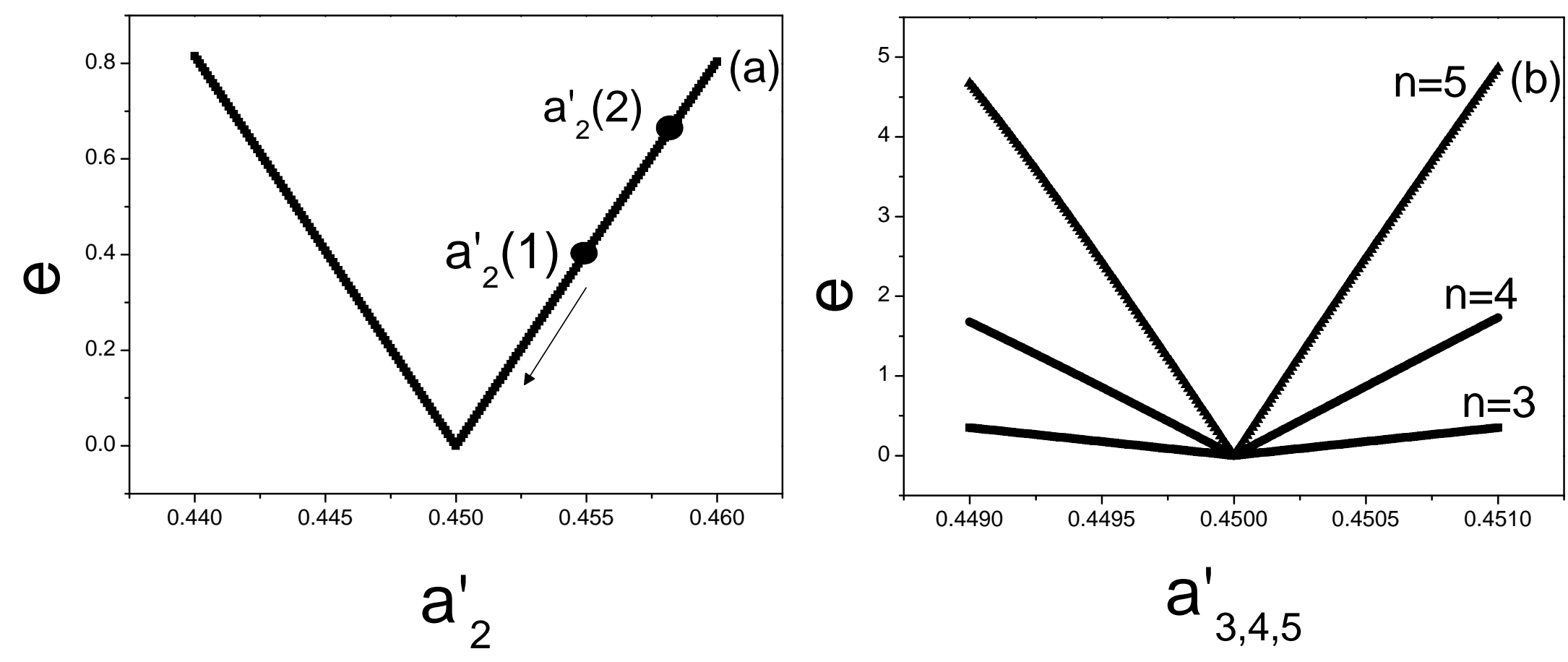

Fig. 1 


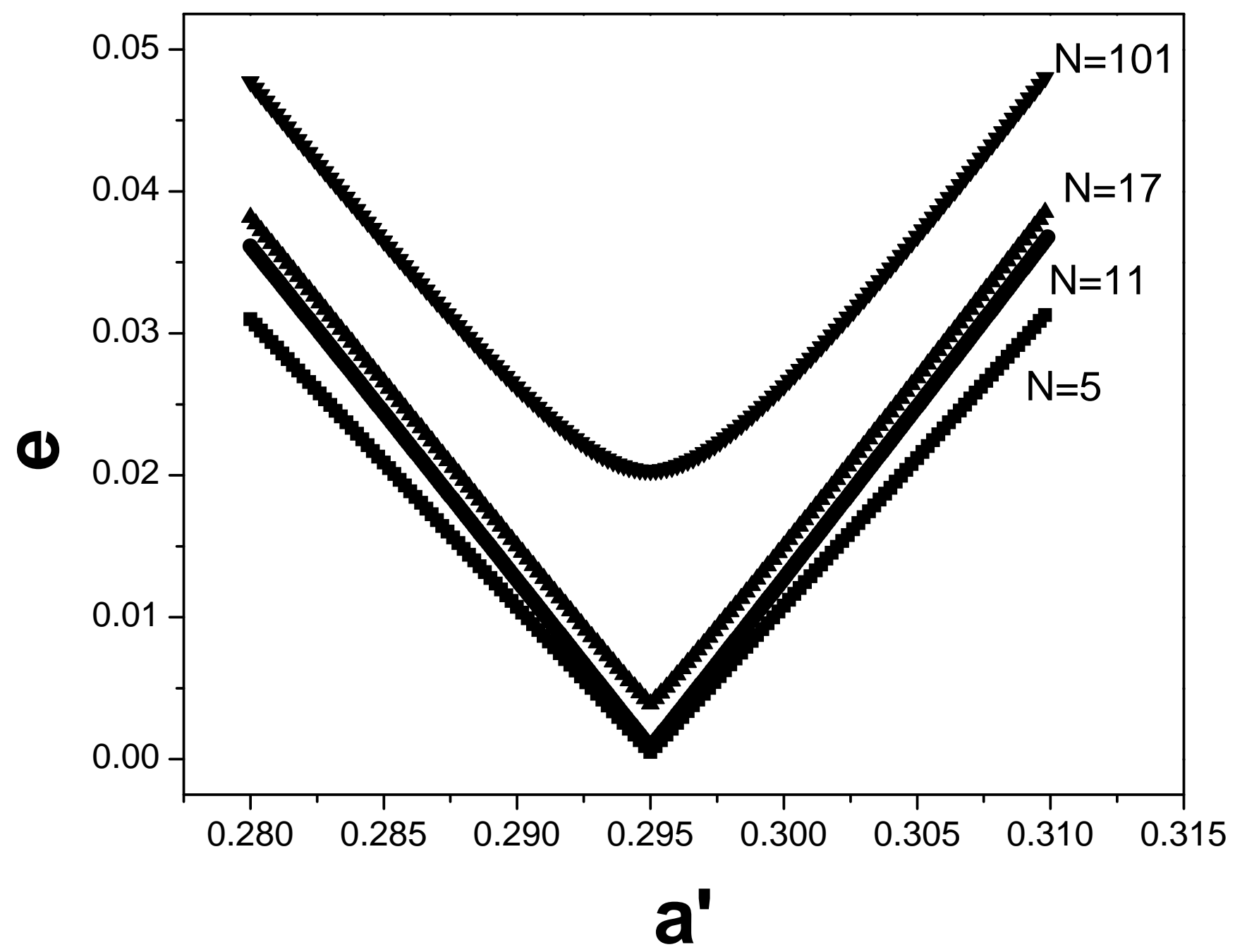

Fig. 2 

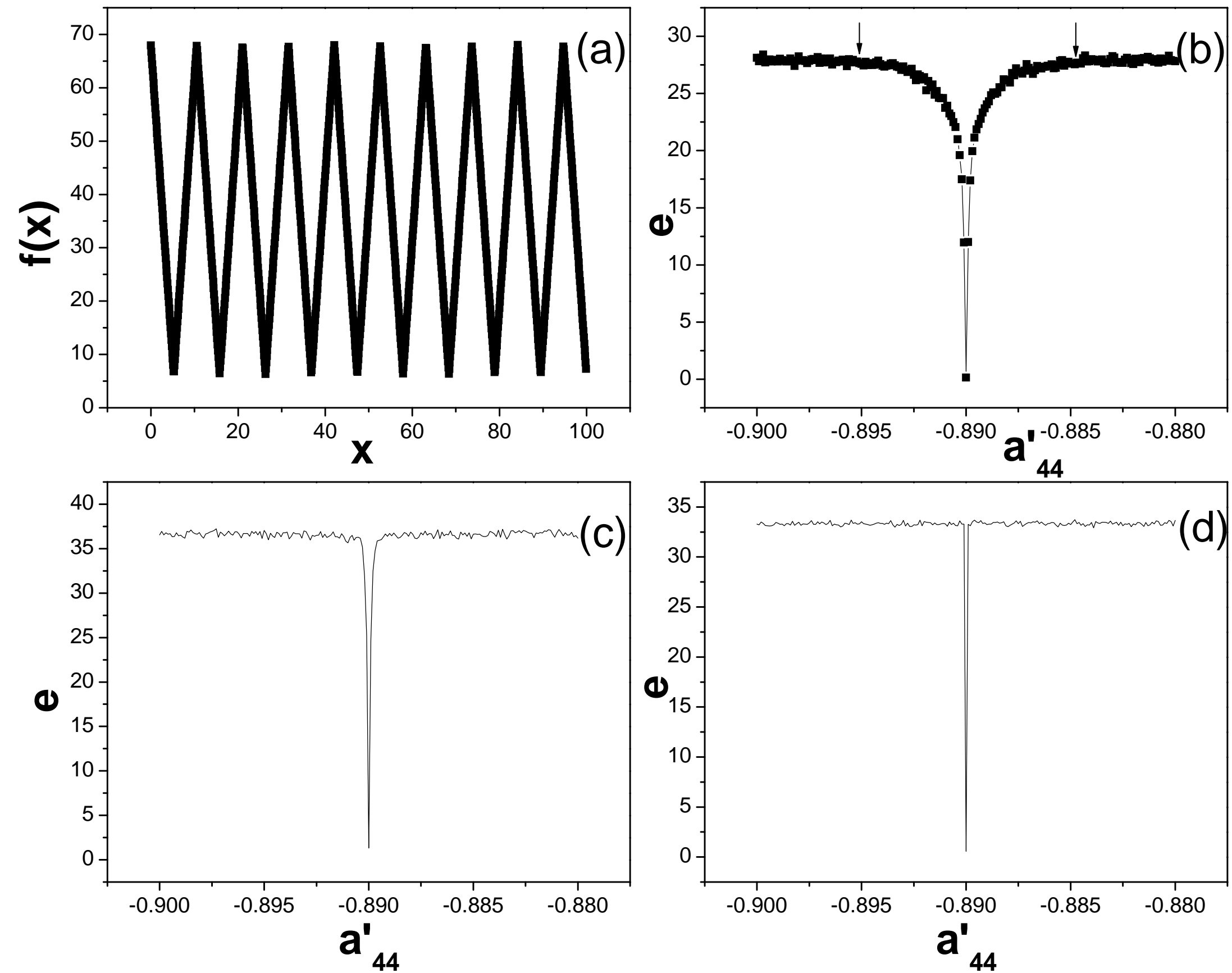

Fig. 3 


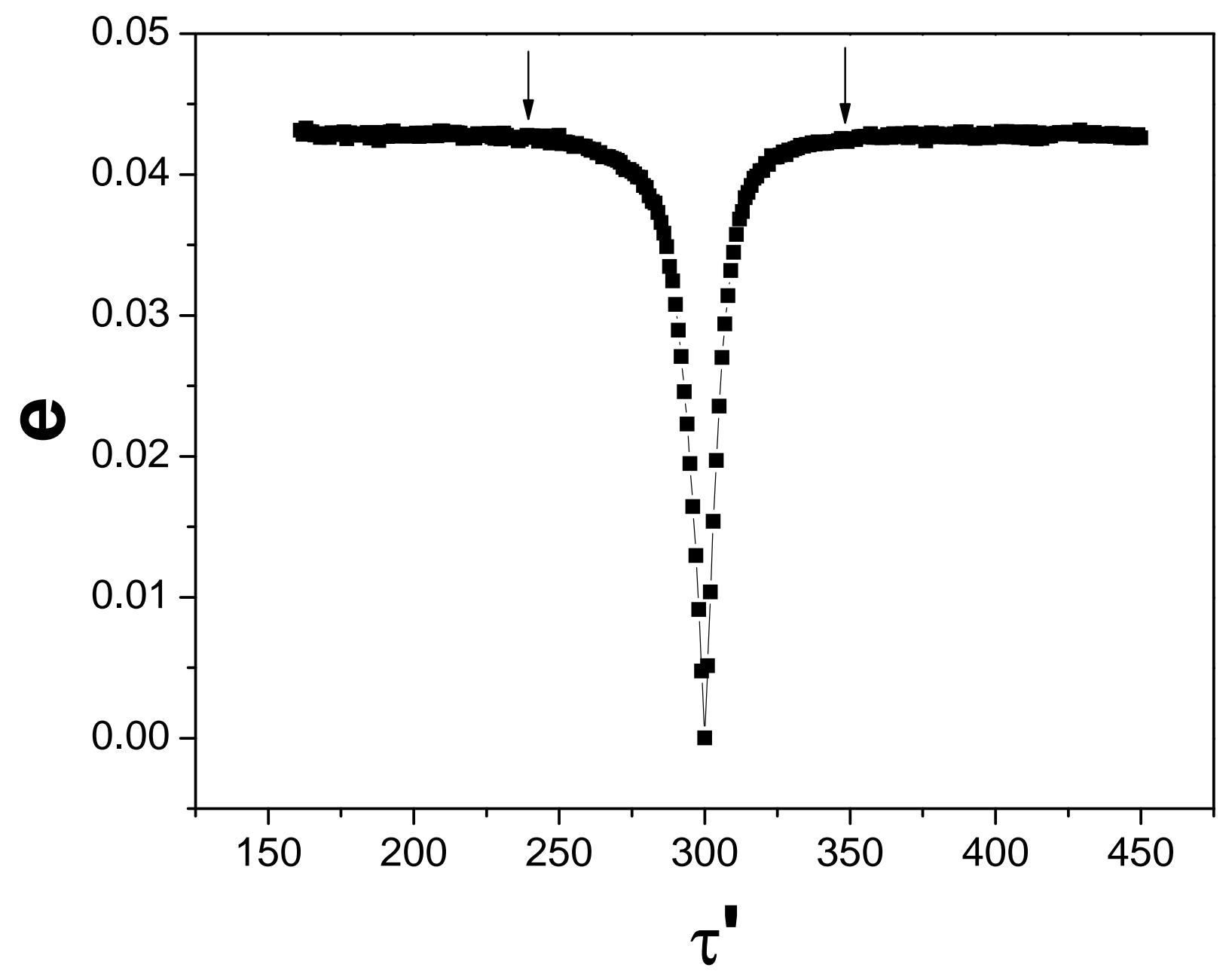

Fig. 4 

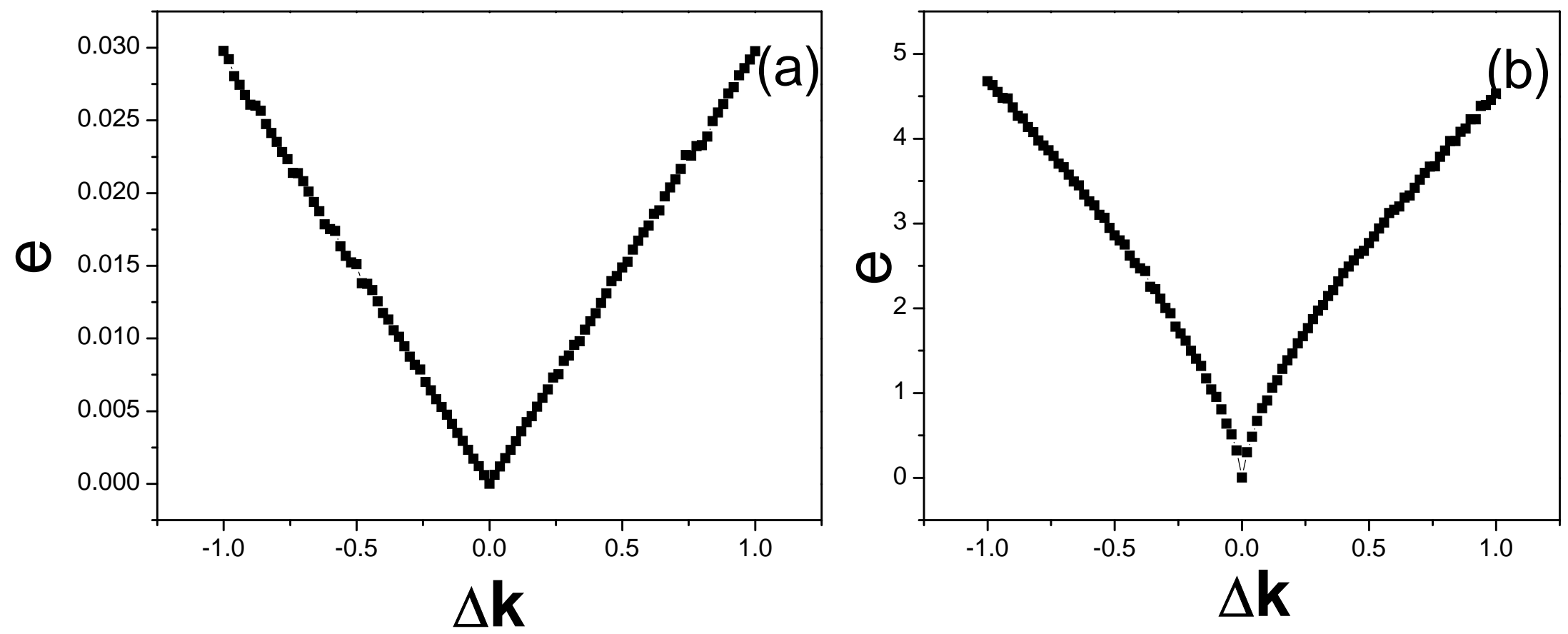

Fig. 5 


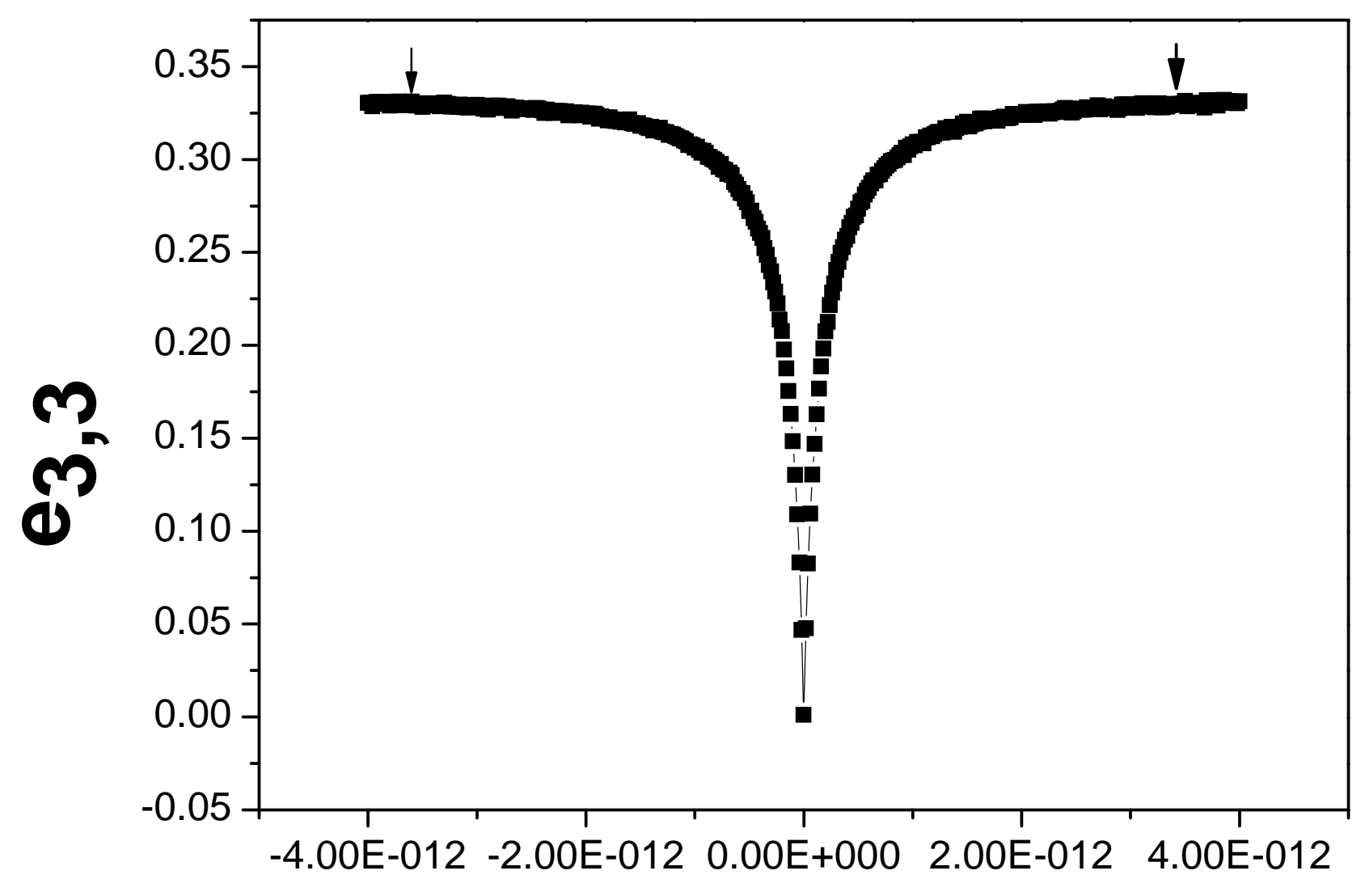

$\Delta \mathbf{a}$

Fig. 6 

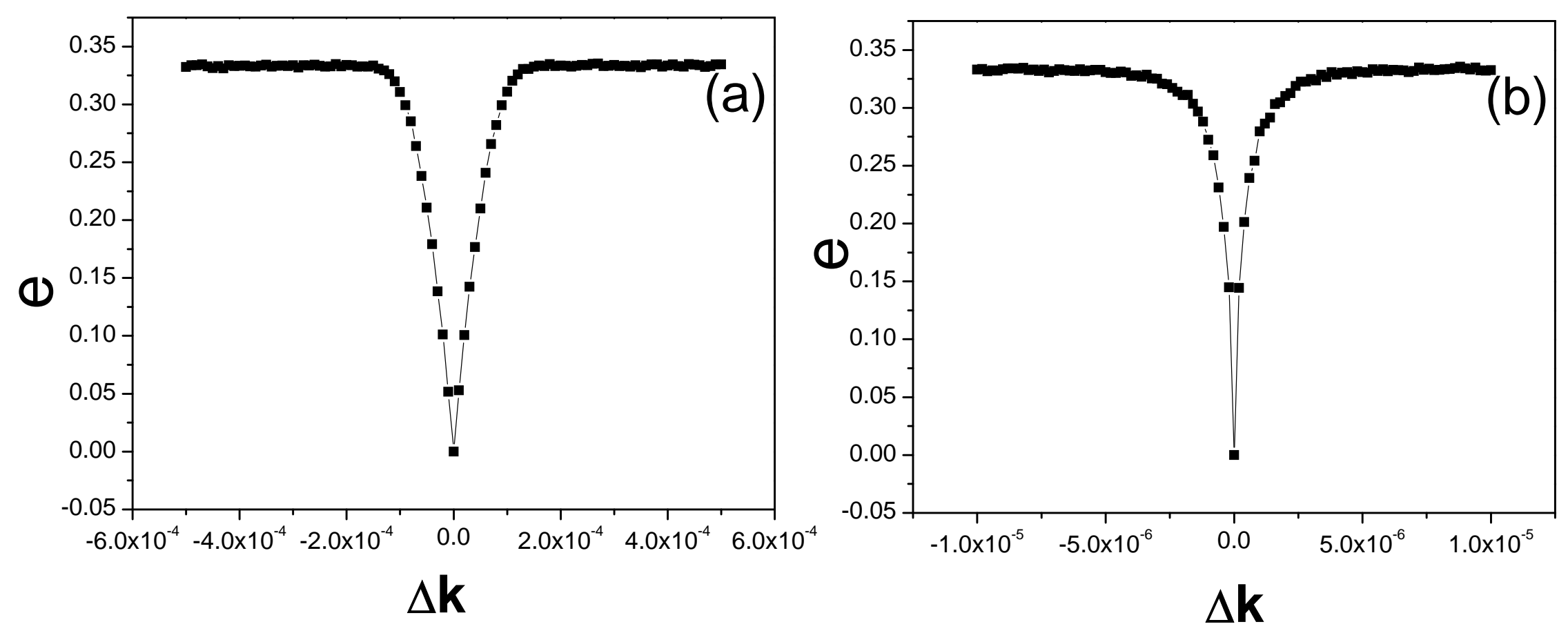

Fig. 7 